\title{
Логика и процедуры поиска вывода ${ }^{1}$
}

\author{
В. Н.Брюшинкин
}

\begin{abstract}
The article deals with the capacity of the proof-search theory for the simulating creativity and solving logico-philosophical problems. The ideas of prominent Russian logician Vladimir Smirnov are analyzed. The proof-search theory emergence is explained in the light of the Boris Gryasnov's model of development of scientific theories. The explanation is based on a new interpretation of unexpected consequences (porism) of Hilbert's proof theory, in particular from the constructing proofs bottom-up in sequential style logical systems, subformula principle and it's interpretation in the field of automatic proof-search procedures.
\end{abstract}

Ключевые слова: логико-математическое доказательство, теория поиска вывода, поризм, аналитическая интерпретация доказательств, принцип подформульности, автоматическое доказательство теорем

$\mathrm{XX}$ век принес сначала в логику, а затем и в философию логики новую тему - проблематику процедур систематического поиска логического вывода. Правда, эта проблематика так или иначе рассматривалась, начиная с Аристотеля, например, при обсуждении проблемы построения силлогизмов: «Теперь следует сказать о том, каким образом мы для данного положения можем всегда иметь достаточно силлогизмов и каким путем мы каждый раз можем найти их начала, ибо надо, пожалуй, не только исследовать, из чего образуются силлогизмы, но и быть в состоянии их строить» $[1$, с. 173]. Аристотель сводит проблему построения силлогизмов к проблеме обнаружения среднего термина. Думаю, что это первая в истории логики попытка построения процедуры поиска логического вывода. Проблематику поиска вывода можно найти и у И. Канта. Так, во Введении к

\footnotetext{
${ }^{1}$ Статья содержит результаты исследований по проекту Российского фонда фундаментальных исследований № 09-06-00092а «Логика И. Канта: реконструкция и современное значение».
} 
«Трансцендентальной диалектике» ставится новая задача по отношению к умозаключениям, решение которой затем окажется парадигматическим по отношению к разуму вообще: «Если, как это нередко случается, вывод задан как суждение, чтобы посмотреть не вытекает ли он из уже данных суждений, посредством которых мыслится совершенно иной предмет, то я ищу в рассудке утверждение этого вывода, а именно не находится ли оно в рассудке при определенных условиях согласно общему правилу» [11, с. 344]. Даже поверхностное знакомство с процедурами поиска вывода, разработанными в современных теориях машинного доказательства теорем, показывает, что здесь ставится не что иное, как задача поиска вывода. Действительно, Кант предполагает наличие суждения $\mathbf{A}$, наличие некоторого множества «данных суждений» $\boldsymbol{\Gamma}$ и спрашивает, имеется ли между ними отношение выводимости: $\boldsymbol{\Gamma} \vdash \mathbf{A}$ ? Это типичная задача поиска вывода. Правда, Кант налагает здесь еще одно ограничительное условие. Посредством посылок вывода должен мыслиться «совершенно иной предмет». Это требование можно на языке современной логики проинтерпретировать как условие, согласно которому множество свободных переменных, встречающихся в посылках, не должно иметь общих элементов с множеством свободных переменных заключения. Или формально: если $\mathbf{V}(\boldsymbol{\Gamma})$ и $\mathbf{V}(\mathbf{A})$ - множества свободных переменных $\boldsymbol{\Gamma}$ и $\mathbf{A}$, соответственно, то $\mathbf{V}(\boldsymbol{\Gamma}) \cap \mathbf{V}(\mathbf{A})=\emptyset$. Иначе говоря, Кант относит к логическому применению разума решение задачи поиска вывода, т.е. фактически расширяет понятие логической прощедуры так, чтобы включить в него не только логические формы понятий, суждений и умозаключений (выводов), но и процедуры поиска вывода (подробнее см. [4]).

Поиск логического вывода (доказательства) до второй половины XX века был неформализованным процессом, о котором высказывались различные эвристические соображения. Отдельным направлением было конструирование логических машин, которые так или иначе также были связаны с построениями логических доказательств. Задача поиска вывода на протяжении тысячелетий относилась к области содержательного мышления, эвристики, способов обнаружения нового знания. В своей статье «Эвристики, алгоритмы и правила логики» О.Ф. Серебрянников 
дает следующее определение эвристики: «Эвристиками мы называем приемы, правила, процедуры, принципы, способствующие организации поиска решения задачи некоторым рациональным или целесообразным путем с практически осуществимыми затратами времени, сил и средств» [14, с. 132]. Существенной особенностью эвристик является то, что стратегия поиска, основанная на эвристиках, не всегда приводит к успеху. Однако даже в случае неудачи эвристического поиска, он должен давать информацию, позволяющую делать дальнейший поиск более эффективным. Существенным вопросом, относящимся к философии логики, является вопрос о том, позволяет ли логика строить формальные модели эвристик или, иначе говоря, являются ли эвристики неформализуемым добавлением к формальным логическим процедурам или имеются формальные логические модели эвристик? Если мы выберем вторую альтернативу, то это будет означать, что нам придется расширить понятие логической процедуры.

В статье В.А. Смирнова «Творчество, открытие и логические методы поиска доказательства» ${ }^{2}$, которая послужила поводом для написания этого текста, ставится задача - «обратить внимание на новые возможности использования логических методов и идей в исследовании исключительно сложной проблематики поиска, открытия и творчества» [16, с. 447]. В этой формулировке выражен ограниченный оптимизм относительно возможностей логики в исследовании эвристических процессов. Вместе с тем обращает на себя внимание то, что Владимир Александрович, который всегда придерживался классических (ФрегеГуссерль) взглядов на логику, доказательство и отношение к процессам порождения нового знания в результате осуществления логических процедур ${ }^{3}$, начинает говорить о возможностях

\footnotetext{
${ }^{2}$ Впервые опубликованной в [15] и перепечатанной в [16]. В дальнейшем будем называть эту статью ТОЛМПД.

${ }^{3}$ В частности, В.А. Смирнов в 70-х годах, когда мы с ним обсуждали эти проблемы, несколько подозрительно отнесся к концепции Я. Хинтикки о приращении информации в ходе логического вывода, основанной на теории дистрибутивных нормальных форм, в силу того, что она расходилась с его основными представлениями о природе логического вывода. Его вполне удовлетворило мое заключение о том, что приращение информации, о котором говорит Хинтикка, происходит не в ходе логического вывода, а в ходе
} 
логики в моделировании эвристических процессов. ТОЛМПД говорит о том, что В.А. к середине 80 -х годов начал рассматривать поиск вывода как логическую процедуру (или как часть ее) и делать на этой основе философские заключения. Чтобы оценить эволюцию взглядов В.А. Смирнова, рассмотрим вкратце эволюцию логики в XIX-XX веках в интересующем нас аспекте.

Основные усилия создателей современной символической логики были направлены на определение независимого от какихлибо внелогических соображений понятия доказательства. С построением исчисления предикатов Готлобом Фреге математическое доказательство стало строгим понятием, определяемым на основе явно введенных логических понятий и структуры логической системы. Таким образом, понятие доказательства опиралось на строго очерченное множество логических истин, определение которых зависело только от явно сформулированных в логической системе отношений и не ссылалось ни на какие внешние для логической системы факторы (например, психологические). Эта установка, сыгравшая решающую роль в становлении символической логики, на долгие десятилетия предопределила интерес логиков к доказательству и связанным с ним понятиям. Эта установка определила характер логических систем и логической проблематики. То, что связано с независимым определением доказательства, есть логика, а все, что не связано с ним, не есть логика. В неклассических логиках, в которых вводились понятия, далеко выходящие за пределы исчисления предикатов, критерием логического характера получавшейся системы были все то же - процедура доказательства, независимая ни от каких внешних факторов. В.А. Смирнов использовал для такого рода первоначальных систем логики, термин «системы фрегерасселовского типа». Такова классическая постановка проблемы отличения логических процедур от нелогических, выработанная на этапе становления математической логики.

В ТОЛМПД Смирнов осмысливает эту классическую поста-

поиска логического вывода. Расхождения у нас были только в вопросе о том, относится ли поиск вывода к логике или поиск вывода носит внелогический характер. Данная статья показывает, что в середине 80-х В.А. начал пересматривать свои взгляды. 
новку вопроса и изменения, которые произошли в ней: «Мне представляется, что необходимо различать три вопроса: что есть доказательство; как искать доказательство; как искать интересующее нас утверждение» [16, с. 438]. Смирнов отмечает, что «логика (дедуктивная, формальная логика) на первых порах ограничивалась исследованием первого вопроса. Основной задачей логики было описание правильных способов рассуждений, т.е. рассуждений, которые при истинности посылок гарантируют истинность заключения» $[16,438-439]^{4}$. Это соответствует тому, что ранее говорилось о роли автономного понятия «доказательство» в системах математической логики. Подводя итог своим рассуждениям, Смирнов замечает, что «имеются четкие и точные критерии, что есть вывод и доказательство» [16, с. 439]. Такие критерии - результат первого этапа становления математической логики. Этот этап завершился знаменитой теоремой Гёделя о неполноте, которая послужила импульсом для становления новой области логики - теории поиска вывода. В некотором смысле можно сказать, что теоремы Гёделя навели на мысль о том, что проблематика теории доказательства не является единственной проблематикой математической логики. В связи с этим рассмотрим подробнее генезис теории поиска вывода в свете новейших методологических исследований.

Б.С. Грязнов предложил модель развития научных теорий, в которой каждая последующая теория возникает как поризм, т.е. непредвиденное следствие из предыдущей теории $T$. В статье «О взаимоотношении проблем и теорий», по существу, содержится оригинальная программа построения теории развития науки, альтернативная программам К. Поппера, Т. Куна, И. Лакатоса. Дело в том, что характерной чертой всех такого рода методологических программ (особенно четко выраженной в концепции К. Поппера) является признание иррациональности возникновения нового знания: научное открытие непредсказуемо и неожиданно, следовательно, оно нелогично и нерационально. В такого рода аргументации всегда имеется скрытая предпосылка, согласно которой в науке нет места теориям, которые

\footnotetext{
${ }^{4}$ Следует обратить внимание на слово «было». Видимо, эту задачу В.А. относит к уже прошедшему этапу развития логики, считая, что теперь у логики новые задачи.
} 
были бы логическими следствиями уже имеющегося знания и вместе с тем были бы непредсказуемы, неожиданны. Б.С. Грязнов опровергает это скрытое допущение: еще античной науке было известно понятие поризма - утверждения, «которое получалось в процессе доказательства теоремы или решения задачи, но получалось как непредвиденное следствие, как промежуточный результат», который, хотя и «получается как логическое следствие, для исследователя. . . может оказаться неожиданным, поскольку не является целью познавательной деятельности» $[9$, c. 114].

Предлагаемая Б.С. Грязновым трактовка научного открытия (возникновения новой теории) как поризма позволяет соединить в рамках одной методологической концепции две, казалось бы, несовместимые черты - логичность (рациональность) и неожиданность (непредсказуемость). Такую концепцию в буквальном смысле можно назвать «Логикой научного открытия». Согласно этой «логике», развитие науки предстает как история решения задач, поставленных внутри имеющихся теорий. Новое знание возникает в ходе решения задач и последующего превращения некоторых из этих решений (поризмов) в новую теорию путем придания им новой интерпретации. Поризм, будучи интерпретирован на новой области объектов, становится новой теорией $T^{*}$. При этом некоторый изоморфный образ теории $T$ при новой интерпретации становится подтеорией $T^{*}$. Указанное понимание развития науки влечет за собой пересмотр многих традиционных представлений, в частности представлений о соотношении проблемы и теории и вообще представлений о задачах методологического исследования.

Эта модель применима и к развитию эмпирических наук, и к развитию математики. Я намереваюсь показать, что модель Грязнова объясняет и развитие логики, в частности, происхождение теории поиска вывода ${ }^{5}$. Теория поиска вывода как теория зародилась в 70-х годах XX века в трудах ленинградского логика и математика С.Ю. Маслова [12]. Вслед за С.Ю. Масловым определим теорию поиска вывода (ТПВ) как область символической логики, занимающуюся обнаружением по исчислению и объекту в языке этого исчисления структуры возможных выво-

\footnotetext{
${ }^{5}$ Впервые этот тезис был высказан в моей работе [3].
} 
дов этого объекта [12, с. 91-92]. Теория поиска вывода в настоящее время вырастает из проблематики автоматического доказательства логических и (или) математических теорем на ЭВМ, ставшей частью более общей проблемы расширения баз знаний в системах «искусственного интеллекта».

В качестве исходной теории $T$ рассмотрим гильбертовскую теорию доказательств, в рамках которой была поставлена задача доказательства непротиворечивости арифметики финитными средствами, заведомо формализуемыми в самой этой арифметике. Традиционный метод проведения доказательств непротиворечивости математических теорий состоял в указании модели рассматриваемой теории, построенной в рамках некоторой теории, непротиворечивость которой не вызывает сомнений. Однако, для теории множеств невозможно построение метатеории, обладающей большей надежностью, чем она сама. Гильберт предложил новый метод обоснования теории множеств, получивший название формалистической программы. Он поставил задачу показать, что применяемые в математике методы доказательства достаточно сильны для того, чтобы получить всю классическую математику, в том числе всю канторовскую теорию множеств, исходя из подходящим образом выбранных аксиом, но не настолько сильны, чтобы вывести из аксиом противоречие. Выполнение этой задачи Гильберт предполагал осуществить в два этапа.

Прежде всего, математика должна быть формализована, то есть необходимо было построить некоторую формальную систему, из аксиом которой с помощью некоторого четко определенного множества правил можно было бы вывести, по крайней мере, основы математики (здесь, прежде всего, имелись в виду арифметика, анализ и теория множеств). Эта формализация идет дальше часто применяемой содержательной аксиоматики, в которой основываются на «содержательно понимаемой» логике и ограничиваются полным перечнем списка всех специальных первоначальных терминов и перечислением всех сформулированных в этих терминах допущений, необходимых для вывода определенной совокупности теорем. Формализация, предполагаемая Гильбертом, не должна была включать в себя никаких неявных значений терминов: во внимание в ней принимаются 
только вид и порядок символов, к последовательностям которых применяются правила вывода. В такой системе проверка того, является ли некоторая цепочка последовательностей символов доказательством последней последовательности в этой цепочке, может быть осуществлена посредством выполнения механических операций.

В качестве второго шага Гильберт намеревался показать, что применение правил вывода к аксиомам никогда не сможет привести к противоречию. Рассуждения, посредством которых доказывается эта метатеорема о невозможности противоречия, должны быть настолько элементарными, чтобы в их справедливости невозможно было усомниться. Все виды рассуждений, применение которых в математике вызывает критику интуиционистов (например, применение закона исключенного третьего к бесконечным множествам), не должны применяться. Эта метатеория, в рамках которой предполагалось исследовать методы математических доказательств, была названа Гильбертом метаматематикой или теорией доказательств. Гильберт настаивал на том, чтобы в теории доказательств разрешалось пользоваться только финитными методами, однако его указания на то, какими свойствами они должны обладать, довольно расплывчаты. Он лишь говорит, что это «...прямые содержательные рассуждения, совершающиеся в виде мысленных экспериментов над наглядно представимыми объектами и не зависящие от предположений аксиоматического характера» [8, с. 59]. Более конкретная характеристика финитным рассуждениям была дана учеником Гильберта Ж. Эрбраном: «под интуиционистским (то есть финитным) рассуждением мы понимаем рассуждение, удовлетворяющее следующим условиям: всегда рассматривается лишь конечное и определенное число предметов и функций; функции эти точно определены, причем определение позволяет произвести однозначное вычисление их значений; никогда не утверждается существование объекта без указания способа построения этого объекта; никогда не рассматривается (как вполне определенное) множество всех предметов $x$ какой-либо бесконечной совокупности; если же говорится, что какое-то рассуждение (или теорема) верно для всех этих $x$, то это означает, что это общее рассуждение можно повторить для каждого конкрет- 
ного $x$, причем само это общее рассуждение следует при этом рассматривать только как образец для проведения таких конкретных рассуждений» (цит. по [17, с. 321]).

Особый интерес представляет методологический анализ этой своеобразной «металогической» теории. Для теории доказательств предметной областью исследования являются реальные доказательства, изобретаемые в содержательных математических теориях. Вместе с тем гильбертовская теория доказательств создает новый объект - формальное доказательство в некотором логико-математическом исчислении. Формальное доказательство в таком случае представляет собой результат абстракции от реальных математических доказательств свойства формальной выводимости теоремы из аксиом теории, представленное в виде синтаксической последовательности формул языка данного исчисления, каждая из которых является либо аксиомой исчисления, либо выводима из предыдущих по явно сформулированным в исчислении правилам вывода. Однако доказательство в логико-математическом исчислении является формальным синтаксическим объектом, а теория доказательств или метаматематика является содержательной теорией о формальных системах. Это означает, что для того, чтобы формальное доказательство стало абстрактным объектом, представляющим объект некоторой теории, оно должно быть интерпретировано, то есть ему должен быть приписан смысл, определяющий место этого объекта в теории. Для Гильберта смысл формальному доказательству придавался его связью с аксиоматическим методом. Аксиоматическое построение теории означает определенную интерпретацию доказательства как движения по нисходящему ряду, от основания к следствию, «сверху вниз», или если вспомнить античную интерпретацию математических доказательств, данную в александрийской математической школе, синтетическое построение доказательства. В.А. Смирнов в ТОЛМПД рассматривает эти смыслы анализа и синтеза, упоминая при этом имена Евклида и Папа Александрийского и опираясь на работу Ф.А. Зеленогорского [10]. Смирнов напрямую связывает понятие анализа с проблематикой поиска вывода, но не упоминает о роли синтеза в применении к теории доказательств. Однако во времена создания гильбертовской тео- 
рии доказательств аксиоматическое построение было единственным известным способом задания логико-математических теорий. Поэтому рассмотрение формального объекта «доказательство» вместе с синтетическим способом его построения «сверху вниз» традиционно воспринималось как нечто само собой разумеющееся. На этом основании можно заключить, что объектом гильбертовской теории доказательств является понятие формального доказательства, которому придана «синтетическая» или «нисходящая» интерпретация.

Невозможность выполнения программы Гильберта в ее первоначальном варианте стала очевидной после того, как Гёдель в 1931 году доказал теорему о неполноте и следствие из этой теоремы (вторую теорему Гёделя), которое показывает невозможность обойтись при обосновании математики только конечными (финитными) методами. После того, как выяснилось, что невозможно доказать формальную непротиворечивость арифметики финитными средствами, возник вопрос, нельзя ли доказать непротиворечивость арифметики с помощью каких-либо методов, не финитных в первоначальном смысле этого слова, но все же достаточно надежно согласующихся с основой концепции формализма. Г. Генцен доказал непротиворечивость арифметики с помощью лишь одного нового метода, выходящего за рамки арифметики в собственном смысле слова, - с помощью так называемой трансфинитной индукции. Для решения задачи доказательства непротиворечивости арифметики Генцен строит сначала натуральные, а затем секвенциальные исчисления. Среди фигур заключения секвенциальных исчислений он выделяет сечение:

$$
\frac{\Gamma \rightarrow \Theta, D \quad D, \Delta \rightarrow \Lambda}{\Gamma, \Delta \rightarrow \Theta, \Lambda}
$$

Все фигуры заключения секвенциальных исчислений, кроме сечения, обладают свойством подформульности - каждая формула из посылки фигуры входит в ее заключение либо сама, либо как подформула более сложной формулы. Для того, чтобы доказать непротиворечивость арифметики, Генцен доказывает основную теорему, говорящую о том, что из исчисления может быть устранено сечение с сохранением множества выводимых в 
исчислении секвенций. При этом он замечает, что в таком исчислении уже все доказательство обладает свойством подформульности - все входящие в него формулы являются подформулами конечной секвенции. Таким образом, свойство подформульности было получено не челенаправленно, а в ходе решения конкретной задачи - доказательства непротиворечивости арифметики, то есть представляет собой поризм. Однако возникновение новой теории на основе поризма требует придания поризму новой интерпретации и включения его в некоторую систему понятий, которая составит ядро новой теории. Обнаружение у доказательств в генценовском секвенциальном исчислении свойства подформульности показало, что доказательство в этом исчислении может строиться как «сверху вниз», так и «снизу вверх». В терминах рассматриваемой модели, это обстоятельство вводит новую аналитическую интерпретацию основного объекта гильбертовской теории доказательств - формального доказательства. Поскольку объект гильбертовской теории доказательств представляет собой понятие формального доказательства вместе с его синтетической интерпретацией «сверху вниз», возможность новой аналитической интерпретации создает возможность нового теоретического объекта. Поскольку же синтетическая и аналитическая интерпретации противоположны, то новый теоретический объект означает рассогласование с первоначальной интерпретацией объекта теории доказательств и требует развития нового взгляда на доказательства в секвенциальных исчислениях как на объекты, обладающие важными «аналитическими» свойствами. Построение доказательства, обладающего свойством подформульности, «снизу вверх» реализует некоторую элементарную стратегию поиска доказательства, в которой структура доказываемой секвенции и выделение в ней главного знака почти однозначно 6 определяют следующий шаг построения доказательства. Тем самым построение доказательства исходной секвенции «снизу вверх» включает в себя формализацию некоторой стратегии обнаружения доказательства, а значит, порождает возможность нового теоретического объекта - поиска

\footnotetext{
${ }^{6}$ За исключением применения так называемых минус-правил: правил введения квантора общности в антецедент и введения квантора существования в сукцедент секвенции.
} 
вывода. Гёдель в своей теореме о неполноте продемонстрировал невыполнимость этой задачи, что привело к попыткам получить доказательство непротиворечивости более сильными (трансфинитными) средствами. Однако оказалось, что аксиоматические системы гильбертовского типа, во-первых, плохо приспособлены к такого рода доказательствам, а, во-вторых, сильно расходятся с теми приемами, которые действительно используются в математической практике. Решая задачу доказательства непротиворечивости арифметики, Генцен в начале 30-х гг. изобретает секвенциальные системы, особенно удобные для такого рода доказательств. В них доказательство непротиворечивости эквивалентно существованию нормальной формы доказательств, в которой более не встречается сечений. Но это автоматически влечет за собой наличие у доказательства свойства подформульности: каждая формула в посылках применения правила является подформулой формул, встречающихся в заключении. Это задает новую точку зрения на доказательства в исчислениях этого типа: «снизу вверх», а эта точка зрения сама по себе подсказывает стратегию поиска вывода. Таким образом, простая перемена точки зрения на выводы в секвенциальных исчислениях ведет к принципиальному сдвигу: от теории доказательств - к теории поиска вывода. Однако этой перемены пришлось ждать примерно еще двадцать лет, и систематически новая точка зрения была проведена только в середине 50-х гг. в семантических таблицах Э. Бета и модельных множествах Я. Хинтикки ${ }^{7}$. В чем дело? Ответ подсказывает модель Грязнова: для превращения непредвиденного следствия (поризма) в новую теорию требуется новая интерпретация с новой предметной областью. Такой интерпретацией послужило автоматическое доказательство теорем на ЭВМ, исходя из потребностей которого, начала систематически развиваться уже собственно теория поиска вывода, ее методы (резолюций, обратный и др.), система и обобщения (до теории творческих, процессов определенного типа - С.Ю. Маслов в $70-\mathrm{х} \mathrm{гг.)}$

Таким образом, свойство подформульности, возникающее как поризм в ходе доказательства непротиворечивости арифмети-

\footnotetext{
${ }^{7}$ Подробнее об аналитической интерпретации доказательств Э. Бетом и Я. Хинтиккой и о том, почему это не привело к формированию ТПВ, см. [7].
} 
ки, вместе с переменой взгляда на работу в исчислении («снизу вверх») и интерпретацией этой работы на новой области автоматического доказательства теорем на ЭВМ породило новую теорию - теорию поиска вывода ${ }^{8}$. Проведенная реконструкция возникновения теории поиска вывода из гильбертовской теории доказательств показывает, что ТПВ стала результатом решения внутрилогических проблем, возникновение которых связано с приложениями логики к основаниям математики. Иначе говоря, возникновение ТПВ показывает, что последняя является логической теорией, формулирующей понятие логической процедуры, представляющей собой расширение традиционного понятия логического доказательства. Логические доказательства становятся частью более обширной системы поиска вывода.

Здесь возникает вопрос о соотношении понятий «метод поиска вывода» и «процедура поиска вывода». Начнем с понятия процедуры. Что же такое логическая процедура и в чем значение этого понятия? Процедура вообще есть последовательность действий, выполняемая по более или менее определенным правилам. Логическую процедуру в первом приближении можно определить как последовательность действий, выполняемых в соответствии с правилами преобразования выражений некоторого естественного или формализованного языка. Однако уже это определение вызывает ряд вопросов: если логическая процедура есть последовательность действий, то: что это за действия? чьи это действия? с чем это действия? - Так вообще пишут по-русски? Возникает задача описания логической процедуры как системы.

Первое, что требуется при системной трактовке логической процедуры, - это осознание того, что логическая процедура является одним из видов познавательных процедур, а, следовательно, имеет субъект-объектную структуру. На мой взгляд, нет необходимости конструировать какой-либо особый «логический» субъект. Субъектом действий, составляющих логическую процедуру, является субъект познания. Специфика субъектобъектных отношений в логических процедурах скорее связана с понятием объекта действий. На первый взгляд, субъект дей-

\footnotetext{
${ }^{8}$ Подробнее объяснение происхождения теории поиска вывода из теории доказательств в рамках поризматической модели см. [18], а также [6].
} 
ствует с формулами или множествами формул языка $L$. Однако являются ли формулы и предложения объектами логических процедур? На мой взгляд, нет, поскольку общезначимые формулы и логически истинные предложения обычно интересуют субъекта не сами по себе, а как выражение отношений между логическими формами высказываний языка $L$. Действительно, вместе с принятием некоторого языка, определением формы его выражений и их семантики фиксируется и множество отношений между логическими формами этих выражений - логических отношений между высказываниями. Эти отношения независимы от субъекта в том смысле, что диктуются, навязываются ему структурой самого языка и его семантикой. Строя выводы, доказательства, опровержения, субъект ставит своей целью обнаружить такие отношения, выраженные формулами или утверждениями о выводимости. Поэтому объектом данной логической процедуры естественно считать некоторое подмножество множества объективных отношений между логическими формами выражений языка $L-$ логических отношений.

При таком понимании субъекта и объекта логических процедур системообразующими связями между компонентами системы - субъектом и объектом - выступают действия субъекта по исследованию объекта - логических отношений.

Логическим аналогом такого рода действий можно считать понятие вывода (доказательства, опровержения). Если определять место логического вывода в системе логической процедуры, то можно сказать, что вывод есть средство фиксации логических отношений, развернутое «изображение» отношения логического следования. Поэтому логический вывод есть результат действий субъекта логических процедур. Сами эти действия - это действия по поиску вывода. Таким образом, на уровне работы с выражениями языка $L$ логическая процедура состоит из двух подсистем: вывода и поиска вывода, которые в совокупности образуют те «внешние действия» (по выражению Гильберта), при помощи которых субъект исследует логические отношения между высказываниями языка $L$.

Рассмотрим, каким образом это понимание логических процедур может быть применено к формализованным логическим системам. С этой точки зрения можно выделить два типа логи- 
ческих систем: во-первых, системы, в которых формализуются понятие общезначимой формулы или отношения следования, и, во-вторых, системы, в которых наряду с формализацией логического следования имеет место хотя бы частичная формализация принципов поиска вывода. Примером систем первого типа могут быть аксиоматические системы гильбертовского типа и системы натурального вывода. Примером систем второго типа могут служить секвенциальные системы.

Можно сказать, что более полная формализация процесса дедуктивного рассуждения происходит в системах второго типа. На основе этих систем можно точно ставить вопрос об эвристических возможностях логических процедур и положительно отвечать на него. В системах первого типа формализуется только подсистема логического вывода, а системные принципы строения логической процедуры не находят полного выражения. В таком случае логические процедуры развертываются как бы в «двух измерениях»: в формальной системе, в которой выписывается вывод, и в «уме» субъекта, работающего с этой формальной системой (ищущего подстановки в аксиомы, изобретающего вспомогательные допущения и т.п.). Системы второго типа - секвенциальные системы кангеровского типа, семантические таблицы Бета и т.п. - частично формализуют и вторую подсистему логических процедур - поиск вывода. Для еще более полной формализации логических процедур, в том виде, в каком они понимаются в настоящей статье, необходима формализация принципов поиска вывода в рассматриваемой логической системе, т.е. формулировка алгоритмов поиска вывода.

Теперь мы можем дать определение логической процедуры: логическая процедура есть последовательность действий суббекта познания с формулами (или множествами формул) формализованного языка (или предложениями естественного язы$\kappa a)$, направленных на обнаружение отношений логических форм высказываний этого языка и выполняемых в соответствии с правилами некоторой логической системы, сформулированной (или подразумеваемой) в этом языке.

Ключевой вопрос, с которым связано рассмотрение важнейших логико-философских проблем ${ }^{9}$, состоит в том, включаем ли

\footnotetext{
${ }^{9}$ Различение логических проблем, философских проблем логики и
} 
мы поиск вывода в понятие логической процедуры, или, иначе говоря, являются ли логическими процедуры поиска логических выводов?

Проведенный анализ понятия логической процедуры показывает, что логическая процедура является целостной системой. Базисный уровень ее представляет собой субъект-объектную структуру, связанную действиями субъекта по исследованию логических отношений; на уровне «внешних действий» (семантики и синтаксиса языка) эта структура проявляется как динамическое взаимодействие двух подсистем: вывода и поиска вывода.

Вообще терминологическое различие «метод поиска вывода»-«процедура поиска вывода» отражает существенное различие в подходе к рассмотрению того, чем занимается логика. При акценте на методы к области логического относятся только сами предписания по осуществлению логических действий, при акценте на процедуры - и само осуществление этих действий. Такое различие порождает и различные подходы в философии логики, в частности, различные решения вопроса о том, возможно ли моделирование логическими структурами процессов мышления или нет.

Тенденцию к включению в состав логических процедур поиска вывода можно заметить и в ТОЛМПД. Однако для В.А. Смирнова этот тезис носит ограниченный характер, поскольку, по мнению Смирнова, если принять этот тезис неограниченно, то возникает опасность проникновения психологизма в логику через «окошко» логических процедур поиска вывода: «Нам важно отметить то, что имеется принципиальная возможность обсуждать проблемы поиска доказательств не в субъективных психологических терминах, а в собственно логических, объективных» [16, с. 442]. Важнейшим философским вопросом здесь является вопрос о том, следует ли включать поиск вывода в структуру логических процедур. Похоже, что В.А. Смирнов склоняется к признанию того, что развитие методов поиска логических выводов во второй половине ХХ века дает нам основание для включения поиска вывода в структуру логических процедур: «Долгое время многие ученые, включая специалистов-логиков, полагали, что задача поиска доказательства является скорее за-

логико-философских проблем см. в [2, с. 7-8]. 
дачей психологической, чем логической. Но развитие логики дало средства для логической постановки проблемы поиска доказательств. Тем самым вторая проблема - как искать доказательство - приобрела характер логической проблемы» $[16$, c. 440]. Однако В.А. Смирнов сразу же ставит следующий вопрос: «В настоящее время логика изучает не только способы рассуждения, но и приходит к изучению процедуры поиска доказательств. Но не означает ли это, что логика вновь возвращается к покинутым ею в свое время позициям психологизма?» [16, c. 443$]$.

Подводя итоги первому этапу развития математической логики, В.А. Смирнов делает совершенно верное наблюдение: «Успехи логики в решении этого основного вопроса (о доказательстве - В.Б.) были обусловлены отказом от психологизма, господствовавшего в логике в ХІХ столетии» [16, с. 439]. Именно психологизм был главным препятствием для формулирования автономного понятия доказательства в логико-математических системах, поскольку основанием доказательств так или иначе считались психологические способности субъекта. Особенно показательной была позиция Д.С. Милля, для которого законы логики представляли собой психологические законы мышления, взятые при определенных ограничениях. Недаром Милль стал объектом критики и Фреге и Гуссерля. По Миллю, (1) логика сводима к психологии, то есть логические понятия могут быть определены в терминах психологических понятий, а логические законы могут быть выведены из психологических законов, (2) логика тривиально является моделью процессов мышления, потому что логика просто является частью этих процессов.

По поводу проблемы психологизма и ТПВ я выскажу некоторые общие соображения, подкрепление которых анализом соотношения конкретных процедур поиска вывода и процессов естественного мышления можно найти в [2].

(1) Конечно, теория поиска вывода не преследует цель изучить, каким образом человек изобретает доказательства, это задача психологии творчества. Однако, думается, не следует отбрасывать возможность того, что, преследуя цель организации наиболее рациональных процедур поиска вывода, логика моделирует некоторые стороны изобретения доказательств чело- 
веком, поскольку человеческие способи поиска вывода одновременно могут оказаться и наиболее рациональными. Последнее утверждение не является верным или неверным a priori. Вопрос о его истинности становится вопросом факта, т.е. сравнения конкретных процедур поиска вывода с процессами естественного мышления.

(2) В ТПВ можно в принципе провести различие между человечески-ориентированными и машинно-ориентированными методами поиска вывода. Такое различие проводится, например, Дж. А. Робинсоном, который к человечески-ориентированным методам поиска вывода относит процедуры поиска вывода, основанные на секвенциальном исчислении и таблицах Бета, а к машинно-ориентированным - метод резолюций [19, с. 169]. Хотя с фактическим проведением такой границы Робинсоном можно не соглашаться, тем не менее различение Робинсона показывает принципиальную возможность проведения такой границы. Иначе говоря, в логике также можно провести грань между методами поиска вывода, более приспособленными для реализации человеком, чем компьютером. В принципе нельзя отвергать возможность сравнения и систематизации методов поиска вывода именно по этому основанию.

(3) Часто говорят о существенном отличии интеллектуальных процедур, разрабатываемых в рамках ИИ, от аналогичных процедур, осуществляемых человеком. Но в современных исследованиях по ИИ этот вопрос не является окончательно решенным. Из них нельзя получить определенного вывода о принципиальной возможности или невозможности моделирования процессов естественного мышления системами ИИ. По-видимому, это также вопрос факта, и решение его зависит от степени успешности систем ИИ, претендующих на моделирование особенностей естественного интеллекта. В силу такой неопределенности в качестве исходного пункта философского исследования можно взять и гипотезу о моделируемости естественного интеллекта искусственным, и гипотезу, запрещающую такое моделирование. Выбор между этими гипотезами в конечном счете будет зависеть от фактического положения дел в области ИИ. Таким образом, мы не можем сказать, что из исследований по ИИ вытекает однозначный антипсихологизм. На мой 
взгляд, большая часть исследований по ИИ склоняется как раз к некоторого рода психологизму, связанному с возможностью моделирования определенных сторон естественного интеллекта в системах ИИ [13].

(4) Логические процедуры не даны логике извне, поскольку логика работает не с самими естественными рассуждениями, а с их представлениями в точных формализованных языках. Но эти представления в некотором (пока не уточняемом) смысле и являются описаниями (точнее сказать, моделями) естественных рассуждений. Если бы перед логикой стояла только задача обоснования методов поиска вывода, то логика, по-видимому, не пошла бы далее процедуры простого перебора (метода Британского музея), поскольку простой перебор является самым обоснованным методом поиска. В силу рекурсивной перечислимости множества теорем исчисления предикатов первого порядка он рано или поздно выдаст вывод любой теоремы этого исчисления.

Однако я думаю, что в настоящее время сам термин «психологизм» (и, соответственно, «антипсихологизм») устарел. В современных исследованиях по философии логики речь уже не идет об обосновании логики со стороны психологии. Речь идет о действительно важном вопросе: является ли логика замкнутой системой теорий, абстрагирующих из систем объективного знания свойство обоснованности высказываний и полностью отделяющих это свойство от тех субъектов, которые изобретают и используют эти обоснования, или логика приносит существенную информацию о человеческом мышлении? Вопрос об обосновании логики психологией снят в результате антипсихологистской критики. Однако остается вопрос о моделировании мышления логическими процедурами. Поэтому для концепций, настаивающих на автономии логики, но утверждающих отношение моделирования между логическими процедурами и определенными аспектами человеческого мышления, больше подходит термин «логический ментализм» ${ }^{10}$. Использование этого термина подчеркивает, что это концепция в философии логики, которая устанавливает объективные отношения между логическим процедурами, совершаемыми в соответствии с правилами той или

\footnotetext{
${ }^{10}$ Я уже обсуждал возможность введения такого термина в [5].
} 
иной формальной системы, и умственными действиями (mental actions) субъекта познания.

Завершить эту статью я хотел бы обширной цитатой из анализируемой работы В.А. Смирнова, намечающей дальнейший путь разработки теории поиска вывода и ее применения как в самой логике, так и в философии логики: «На протяжении последних ста лет своего развития логика ограничивала свои задачи процедурами доказательства, не претендуя на исследование методов открытия, на исследование процессов творчества. В этом ограничении своих задач был залог многих ее успехов. Однако к настоящему времени разработаны средства, позволяющие исследовать процедуры поиска доказательств и их индуктивного подтверждения. Запросы практики, особенно задачи создания „искусственного интеллекта“, настоятельно требуют разработки вопросов, относящихся к проблемам выдвижения и выбора гипотез, индуктивных закономерностей. Логическому подходу к этим проблемам будет принадлежать важное место» [16, с. 447].

\section{Литература}

[1] Аристотель. Первая аналитика // Сочинения в 4-х томах. М.: Мысль, 1978. T. 2. C. 173 .

[2] Брюшинкин В.Н. Логика, мышление, информация. Л.: Изд-во ЛГУ, 1988.

[3] Брюшинкин В.Н. О возникновении теорий в логике: теория поиска вывода как поризм // Современная логика: Проблемы теории, истории и применения в науке: Сб. науч. тр. / Под. ред. А.Я Слинина. Л.: Изд-во Ленинградского унта, 1990. С.17-18.

[4] Брюшинкин В.Н. Метапсихологизм Канта // Кантовский сборник. Вып. 24. Калининград: Изд-во КГУ, 2004. С. 65-73.

[5] Брюшинкин В.Н. Антропологические измерения логики // Вестник Российского государственного университета имени Иммануила Канта. Вып. 6. Сер. Гуманитарные науки. Калининград: Изд-во РГУ им. И Канта, 2006. С. 6-11.

[6] Брюшинкин B.Н., Ходикова Н.А. Рациональная реконструкция происхождения теории поиска вывода из гильбертовской теории доказательств // Модели рассуждений - 1: Логика и аргументация: Сб. науч. ст. / Под общ. ред. В.Н. Брюшинкина. Калининград: Изд-во РГУ им. И. Канта, 2007. С. 205-219.

[7] Брюшинкин B.H., Ходикова Н.A. Аналитическая интерпретация доказательств в таблицах Бета и модельных множествах Хинтикки // Аргументация и интерпретация. Исследования по логике, аргументации и истории философии: Сб. научн. статей. / Под ред. В.Н. Брюшинкина. Калининград: Изд-во РГУ им. И. Канта, 2006. С. 39-46.

[8] Гилъберт Д., Бернайс П. Основания математики. Логические исчисления и формализация арифметики. М.: Наука, 1979. С. 59.

[9] Грязнов Б.С. Логика, рациональность, творчество. М.: Наука, 1982. С. 114.

[10] Зеленогорский Ф.А. О математическом, метафизическом, индуктивном и критическом методах исследования и доказательства. Харьков, 1877. 
[11] Кант И. Критика чистого разума // Кант И. Соч. в шести тт. М.: Мысль, 1966. T. 3. C. 344

[12] Маслов С.Ю. Теория дедуктивных систем и ее применения. М.: Радио и связь, 1986.

[13] Поспелов Д.А. Моделирование рассуждений. Опыт анализа мыслительных актов. М.: Радио и связь, 1989.

[14] Серебрянников О.Ф. Эвристики, алгоритмы и правила логики // Проблемы законов науки и логики научного познания: Сб. науч. тр. / Под ред. И.Я. Чупахина, В.П.Рожина. Л.: Изд-во Ленинградского университета, 1980. С. 132.

[15] Смирнов B.A. Творчество, открытие и логические методы поиска доказательства // Природа научного открытия. М.: Наука., 1986. С. 101-114.

[16] Смирнов B.A. Творчество, открытие и логические методы поиска доказательства // Логико-философские труды В.А. Смирнова / Под ред. В.И. Шалака, М.: Эдиториал УРСС, 2001. С. 438-447.

[17] Френкель А., Бар-Хиллел И. Основания теории множеств. М.: Мир, 1966. C. 321.

[18] Ходикова Н.А. Логико-методологическое исследование происхождения теории поиска вывода. Диссертация на соискание ученой степени кандидата философских наук. Калининград, 2004

[19] Robinson J.A. Logic: form and function. Edinburg, 1979. P. 169. 